\title{
Adaptive Robust Simultaneous Stabilization of Two Dynamic Positioning Vessels Based on a Port-Controlled Hamiltonian (PCH) Model
}

\author{
Pei Zhou ${ }^{D}$, Renming Yang * ${ }^{D}$, Guangyuan Zhang and Yaozhen Han \\ School of Information Science and Electrical Engineering, Shandong Jiaotong University, Jinan 250357, China; \\ 17021006@stu.sdjtu.edu.cn (P.Z.); xdzhanggy@163.com (G.Z.); hyz125@163.com (Y.H.) \\ * Correspondence: renmingyang0222@163.com; Tel.: +86-183-9682-8269
}

Received: 12 September 2019; Accepted: 14 October 2019; Published: 17 October 2019

check for updates

\begin{abstract}
In this paper, the adaptive robust simultaneous stabilization problem of two ships is studied. Firstly, the water surface three-degree-of-freedom ship models are transformed into port-controlled Hamiltonian (PCH) models. Using a single output feedback controller, the two PCH systems are combined to generate an enhanced PCH system based on Hamiltonian structural attributes. Then, considering the situation with both external interference and structural parameter perturbation in the systems, an adaptive robust output feedback controller is designed to stabilize the two systems simultaneously. Finally, the effectiveness of the controller proposed in this paper is illustrated by a simulation example.
\end{abstract}

Keywords: dynamic positioning ship; adaptive robust simultaneous stabilization; port controlled Hamilton model

\section{Introduction}

Dynamic positioning (DP) refers to maintaining an ocean vehicle on the surface of the sea at an accurate positioning by using its own power system and various propellers installed independently without the aid of a mooring system [1]. DP is widely used in the exploration and exploitation of marine resources such as oil and gas in deep-sea areas, salvage vessels, fire boats and so on, and is the key to maintaining the normal operation of floating platforms and related ships in marine development.

In the 1960s, the first generation of dynamic positioning products appeared, which adopted classical control theory to design the controller, i.e., a conventional proportion integral derivative (PID) controller. In the mid-1970s, Balchen et al. proposed a dynamic positioning control method based on modern control theory, i.e., the second generation dynamic positioning system, which combines optimal control with Kalman filtering theory. In recent years, the third generation dynamic positioning system has adopted the theory and method of intelligent control, which makes the dynamic positioning control further develop toward the direction of intellectualization. These intelligent control methods are mainly embodied in robust control, fuzzy control, non-linear model predictive control and so on [2].

At present, dynamic positioning technology plays an extremely important role in the process of ocean development and research. Especially, with the continuous development of marine techniques, the range of people's activities gradually extends from shallow sea to the deep sea, which puts forward higher requirements for the efficiency of marine operations and the automation of marine equipment. As a result, the coordinated formation control of multiple dynamic positioning vessels emerges [3]. As is well known, besides the advantages of improving operation efficiency and reducing cost, the redundancy of multi-dynamic positioning ship formation can also improve the robustness of the whole formation system [4]. Moreover, the multi-ship formation system has the advantage 
of avoiding being life-threatening for operators when performing dangerous tasks, and is easy to expand and upgrade. Therefore, the multi-ship formation cooperative control problem has become an important research direction and has attracted the attention of many scholars [5]. However, due to the complexity of ship motion and the strong disturbance of the marine environment, it is a very difficult task to study the formation control of multiple dynamic positioning ships. Although the research on multi-agent formation control has made considerable progress in recent decades [6,7], it should be pointed out that, on the one hand, most of the existing research results are based on linear systems or systems with linear main parts, there are few research results on the non-linear systems; and on the other hand, in view of the complexity of the non-linear system and the challenge of simultaneous stabilization problem, fewer results are obtained for multi-dynamic positioning ship systems, except [4]. In [4], the authors designed a coordinated formation controller, based on the control theory of artificial potential function, combined with the adaptive backstepping sliding mode control approach and Lyapunov function method. Compared with the Hamilton method, this method is not direct enough and the design process is more complicated. This paper attempts to apply the Hamilton method to ship dynamic positioning, studies the simultaneous stabilization of two ships, and develops some concise results.

In this paper, the Hamilton function method is proposed to study the simultaneous stabilization problem of two ships' dynamic positioning and the simultaneous stabilization; adaptive simultaneous stabilization, and adaptive robust simultaneous stabilization conditions are established, and the corresponding simultaneous stabilization controllers are designed. Firstly, the two water surface three-degree-of-freedom ship models are transformed into an equivalent port-controlled Hamiltonian (PCH) model. Then, based on the extended dimension technology, an extended Hamiltonian system is constructed and the problems of simultaneous stabilization, adaptive simultaneous stabilization and adaptive robust simultaneous stabilization are studied, and certain corresponding results are obtained. Finally, a simulation example shows the effectiveness of the designed controller.

The main contributions of this paper are as follows: (1) In contrast to the existing literature, [8-11], the present paper investigates two ships and develops several simultaneous stabilization results. While in [8-11], the authors studied a single ship's stabilization problem rather than the simultaneous stabilization one. (2) Unlike recent literature [12-17] on the simultaneous stabilization problem, the present paper studies the dynamic positioning problem of two ships, and develops some simultaneous stabilization, robust simultaneous stabilization, and adaptive robust simultaneous stabilization results based on the PCH model method. In [12,13,15-17], the papers developed some simultaneous stabilization results for some nonlinear systems by applying the linear approximation method, rather than for ship systems respectively. In [12], the authors mainly applied the state space method and the control Lyapunov function method to study the simultaneous stabilization problem of a set of uncertain nonlinear systems, obtain a simultaneous stabilization feedback controller. In [13], based on a common control storage function, a continuous state feedback law was designed to simultaneously $H_{\infty}$ stabilize all the closed-loop systems. In [15-17], by using the Lyapunov-Krasovskii functional and matrix inequality methods, the authors designed corresponding feedback controllers to study the simultaneous stabilization control problem for a class of nonlinear time-delay systems. In addition, in [14], although the authors studied the simultaneous stabilization problem of a class of nonlinear port-controlled Hamiltonian systems, they only obtained some theoretical results on the Hamiltonian system rather than an actual ship system.

Notation: $\mathbb{R}$ is the set of real numbers. $\mathbb{R}^{n}$ denotes the n-dimensional Euclidean space. $\mathbb{R}^{n \times l}$ is the real matrices with dimension $n \times l$. The notation $R(x) \geq 0(R(x)>0)$ means that the matrix $R(x)$ is positive semi-definite (positive definite). For convenience, we denote $\frac{\partial H(x)}{\partial x}$ by $\nabla H(x) . L_{2}$ denotes the space of square integrable signals with norm defined by $\|x\|_{2}=\left(\int_{0}^{\infty}\|x(t)\|^{2} d t\right)^{\frac{1}{2}}$, where $\|x(t)\|$ denotes the Euclidean norm on $\mathbb{R}^{n}$. 


\section{Problem Formulation and Preliminaries}

This section gives a number of lemmas and preparation works.

\subsection{Some Lemmas}

Consider the the system [18]:

$$
\dot{x}=f(x)+g_{1}(x) u+g_{2}(x) \omega,
$$

where, $x \in \mathbb{R}^{n}$ is the state, $u \in \mathbb{R}^{m}$ is the input, $\omega \in \mathbb{R}^{s}$ is the interference, $g_{1}(x), g_{2}(x)$ is the weight matrices of the appropriate dimensions.

The $L_{2}$ interference suppression problem is described as follows: Give a penalty signal $z=q(x)$ and interference suppression level $\gamma>0$, find a feedback control law $u=k(x)$ and a positive definite storage function $V(x)$ such that the following $\gamma$ - dissipative inequality holds along the trajectory of the closed-loop system:

$$
\dot{V}+Q(x) \leq \frac{1}{2}\left\{\gamma^{2}\|\omega\|^{2}-\|z\|^{2}\right\}, \forall \omega \in L_{2}
$$

where, $Q(x)$ is a non-negative function.

Remark 1. From the inequality (2), it is easy to obtain the following characteristics:

$P 1$. The $L_{2}$ gain from $\omega$ to $z$ is less than $\gamma$.

$P 2$. When $\omega=0$, the closed loop system is Lyapunov stable. Further, if $Q(x) \neq 0(\forall x \neq 0)$, the closed loop system is asymptotically stable.

Under the above definition, we give a judgment method for the $L_{2}$ interference suppression problem of the generalized Hamiltonian system.

Consider the following generalized Hamiltonian system:

$$
\left\{\begin{array}{l}
\dot{x}=[J(x)-R(x)] \nabla H+g_{1}(x) u+g_{2}(x) \omega, \\
z=h(x) g_{1}^{T}(x) \nabla H,
\end{array}\right.
$$

where, $R(x) \geq 0$, if $x \neq 0, H(x)>0$ is Hamiltonian function of the system, $h(x)$ is a weight matrix, $\nabla H$ is the gradient vector of $H(x)$.

Giving interference suppression level $\gamma>0$, and supposing $z=h(x) g_{1}^{T}(x) \nabla H$, then we have the following conclusion.

Lemma 1 ([18]). Consider the system (3). Assuming that the interference suppression level $\gamma>0$ is given, if

$$
R(x)+\frac{1}{2 \gamma^{2}}\left[g_{1}(x) g_{1}^{T}(x)-g_{2}(x) g_{2}^{T}(x)\right] \geq 0
$$

holds and the control laws is designed as:

$$
u=-\left[\frac{1}{2} h^{T}(x) h(x)+\frac{1}{2 \gamma^{2}} I_{m}\right] g_{1}^{T}(x) \nabla H
$$

then the closed loop system consisting of Equations (3) and (5) satisfies the following dissipative inequality:

$$
\dot{H}+d H\left[R-\frac{1}{2 \gamma^{2}}\left(g_{2}(x) g_{2}^{T}(x)-g_{1}(x) g_{1}^{T}(x)\right)\right] \nabla H \leq \frac{1}{2}\left\{\gamma^{2}\|\omega\|^{2}-\|z\|^{2}\right\} .
$$

where $Q(x)=d H\left[R-\frac{1}{2 \gamma^{2}}\left(g_{2}(x) g_{2}^{T}(x)-g_{1}(x) g_{1}^{T}(x)\right)\right] \nabla H$. 


\subsection{Dynamic Model of Ship}

The nonlinear mathematical model of ship dynamic positioning studied in this paper is described as [19]:

$$
\left\{\begin{aligned}
\dot{\eta} & =R(\psi) v \\
M \dot{v} & =-D v+\tau+\omega
\end{aligned}\right.
$$

where $\eta=[n, e, \psi]^{T} \in \mathbb{R}^{3 \times 1}$ is the position vector of the ship in the northeastern coordinate system, and denotes the ship's surge displacement, sway displacement and yaw angle respectively. $v=[\sigma, \mu, r]^{T} \in \mathbb{R}^{3 \times 1}$ is the velocity vector in the ship coordinate system, which represents surge speed, sway speed and yaw angular speed, respectively. $\tau \in \mathbb{R}^{3 \times 1}$ represents the total moment, $0<M \in \mathbb{R}^{3 \times 3}$ is the additional mass matrix of the hull, $0<D \in \mathbb{R}^{3 \times 3}$ is the damping matrix coefficient, $\omega$ is an unknown disturbance term, which represents the comprehensive constant interference force and moment generated by the environmental factors such as wind, wave and flow. $R(\psi) \in \mathbb{R}^{3 \times 3}$ is a coordinate transformation matrix satisfying $R(\psi)=\left[\begin{array}{ccc}\cos \psi & -\sin \psi & 0 \\ \sin \psi & \cos \psi & 0 \\ 0 & 0 & 1\end{array}\right]$ and $R^{-1}(\psi)=R^{T}(\psi)$.

In this paper, the Hamilton method is used to study the simultaneous stabilization problem of the ship. It is necessary to convert the ship model (7) into a port-controlled Hamilton model. To this end, in the following, we introduce some related content on the Hamilton system.

$$
\begin{gathered}
\dot{x}=[J(x)-R(x)] \nabla H(x)+g(x) u, \\
y=g^{T}(x) \nabla H(x),
\end{gathered}
$$

where $x \in \mathbb{R}^{n}$ is a state variable, $H: \mathbb{R}^{n} \rightarrow \mathbb{R}$ is a scalar function of state variables, called a Hamilton function, which represents the total energy of the system. $u, y \in \mathbb{R}^{m}$ are input-output vectors. $J(x) \in \mathbb{R}^{n \times n}$ is an skew matrix, which describes no energy loss of the system (8), $R(x)\left(\in \mathbb{R}^{n \times n}\right) \geq 0$ is a symmetric matrix, expresses the energy dissipation part of the system. $g(x) \in \mathbb{R}^{n \times m}$ is a weight matrix.

Next, we give the process of converting system (7) into its Hamilton form.

Define ship position error vector $x_{1}$ as

$$
x_{1}=\eta-\eta_{d}
$$

where $\eta_{d}=\left[n_{d}, e_{d}, \psi_{d}\right]^{T}$ is the vector of the ship's desired position and yaw angle. Thus, from the Equations (7) and (10), we have

$$
\dot{x_{1}}=R(\psi) v
$$

According to the traditional inversion method, defining the ship's speed error vector $x_{2} \in \mathbb{R}^{3}$, then one can obtain

$$
x_{2}=v-\alpha_{1}
$$

where $\alpha_{1} \in \mathbb{R}^{3}$ satisfying

$$
\alpha_{1}=R^{-1}(\psi)\left(-k_{1} x_{1}\right),
$$

and $k_{1} \in \mathbb{R}^{3 \times 3}$ is the positive definite parameter diagonal matrix. 
Derivative on both sides of Equation (12):

$$
\dot{v}=\dot{x}_{2}+\dot{\alpha}{ }_{1}
$$

Substituting (10-14) into (7), we have

$$
\left\{\begin{array}{l}
\dot{x_{1}}=R(\psi) x_{2}-k_{1} x_{1} \\
\dot{x_{2}}=-M^{-1} D x_{2}+M^{-1} D R^{-1}(\psi) k_{1} x_{1}+M^{-1} \tau+M^{-1} \omega-\dot{\alpha_{1}} .
\end{array}\right.
$$

Design $\tau$ as follows:

$$
\tau=D \alpha_{1}+M \dot{\alpha_{1}}-M R^{T}(\psi) x_{1}+u
$$

where $u$ is a new input, from which and the system (15), we obtain

$$
\left\{\begin{array}{l}
\dot{x_{1}}=R(\psi) x_{2}-k_{1} x_{1} \\
\dot{x_{2}}=-R^{T}(\psi) x_{1}-M^{-1} D x_{2}+M^{-1} u+M^{-1} \omega .
\end{array}\right.
$$

Furthermore, the system (17) is expressed as the following Hamiltonian form:

$$
\dot{x}=[J(x)-R(x)] \nabla x H(x)+g(x) u+\widetilde{g}(x) \omega,
$$

$$
y=g^{T}(x) \nabla H(x),
$$

where, $J(x)=\left[\begin{array}{cc}0 & R(\psi) \\ -R^{T}(\psi) & 0\end{array}\right], R(x)=\left[\begin{array}{cc}k_{1} & 0 \\ 0 & M^{-1} D\end{array}\right], g(x)=\widetilde{g}(x)=\left[\begin{array}{c}0 \\ M^{-1}\end{array}\right]$, $H(x)=\frac{1}{2} \sum_{i=1}^{3}\left(x_{1 i}^{2}\right)+\frac{1}{2} \sum_{i=1}^{3}\left(x_{2 i}^{2}\right)$.

Obviously, system (17) is equivalent to system (18). In the next section, we will study simultaneous stabilization problem of the system (18).

\section{Adaptive Robust Simultaneous Stabilization}

In the section, we firstly present a simultaneous stabilization result and an adaptive simultaneous stabilization result for the systems without the disturbance, and then we study the adaptive robust simultaneous stabilization problem on the system with the external disturbance, and an output feedback robust controller is designed.

\subsection{Simultaneous Stabilization}

Based on system (18) and system (19), consider the following two PCH systems with $\omega=0$ as:

$$
\begin{aligned}
& \Sigma_{1}:\left\{\begin{array}{l}
\dot{x}=\left[J_{1}(x)-R_{1}(x)\right] \nabla H_{1}(x)+g_{1}(x) u, \\
y_{1}=g_{1}^{T}(x) \nabla H_{1}(x),
\end{array}\right. \\
& \Sigma_{2}:\left\{\begin{array}{c}
\dot{\xi}=\left[J_{2}(\xi)-R_{2}(\xi)\right] \nabla H_{2}(\xi)+g_{2}(\xi) u, \\
y_{2}=g_{2}^{T}(\xi) \nabla H_{2}(\xi),
\end{array}\right.
\end{aligned}
$$

where $x, \xi$ and $y_{1}, y_{2}$ are the states and outputs of the two systems, respectively; $u$ is the control input; $H_{i}(x)$ is the Hamiltonian function with a local strict minimum at $x_{e}^{(i)}, i=1,2$, and $x_{e}^{(1)}=x_{0}, x_{e}^{(2)}=\xi_{0}$. Note: $J_{i}(x)=\left[\begin{array}{cc}0 & R_{i}(\psi) \\ -R_{i}^{T}(\psi) & 0\end{array}\right]=-J_{i}^{T}(x), R_{i}(x)=\left[\begin{array}{cc}k_{1} & 0 \\ 0 & M_{i}^{-1} D_{i}\end{array}\right], g_{i}(x)=\left[\begin{array}{c}0 \\ M_{i}^{-1}\end{array}\right], i=1,2$. 
Obviously, when $u=0$, both systems (20) and (21) are stable, but not asymptotically stable.

We design an output feedback law $u=u\left(y_{1}, y_{2}\right)$, so that under the control law, the systems (20) and (21) can be asymptotically stable at the same time.

Now, we propose the main result.

Theorem 1. Assume that there exists a symmetric matrix $K \in \mathbb{R}^{6 \times 6}$ such that

$$
\left\{\begin{array}{l}
R_{1}(x)+K_{11}(x, x)>0 \\
R_{2}(\xi)-K_{22}(\xi, \xi)>0
\end{array}\right.
$$

where,

$$
K_{i j}(x, \xi)=g_{i}(x) K g_{j}^{T}(\xi),(i, j=1,2) .
$$

Then, the output feedback controller

$$
u=-K\left(y_{1}-y_{2}\right),
$$

can simultaneously stabilize systems (20) and (21).

Proof. Substituting (24) into systems (20) and (21), we obtain

$$
\left\{\begin{array}{l}
\dot{x}=\left[J_{1}(x)-R_{1}(x)\right] \nabla H_{1}(x)-g_{1}(x) K g_{1}^{T}(x) \nabla H_{1}(x)+g_{1}(x) K g_{2}^{T}(\xi) \nabla H_{2}(\xi), \\
\dot{\xi}=\left[J_{2}(\xi)-R_{2}(\xi)\right] \nabla H_{2}(\xi)-g_{2}(\xi) K g_{1}^{T}(x) \nabla H_{1}(x)+g_{2}(\xi) K g_{2}^{T}(\xi) \nabla H_{2}(\xi),
\end{array}\right.
$$

from which, the above system can be expressed further as

$$
\dot{X}=[J(X)-R(X)] \nabla H(X),
$$

where,

$$
\begin{gathered}
X=\left[x^{T}, \xi^{T}\right]^{T}, H(X)=H_{1}(x)+H_{2}(\xi), \nabla H(X)=\left[\begin{array}{l}
\nabla H_{1}(x) \\
\nabla H_{2}(\xi)
\end{array}\right], \\
J(X)=\left[\begin{array}{cc}
J_{1}(x) & K_{12}(x, \xi) \\
-K_{12}^{T}(x, \xi) & J_{2}(\xi)
\end{array}\right], \\
R(X)=\left[\begin{array}{cc}
R_{1}(x)+K_{11}(x, x) & 0 \\
0 & R_{2}(\xi)-K_{22}(\xi, \xi)
\end{array}\right] .
\end{gathered}
$$

Obviously, $J(X)$ is a skew matrix, and from (22), $R(X)$ is positive definite. Thus, the system (26) is an augmented strictly dissipative Hamiltonian system.

Let $X_{0}:=\left[x_{0}^{T}, \xi_{0}^{T}\right]^{T}$. Since $\nabla H_{1}\left(x_{0}\right)=0, \nabla H_{2}\left(\xi_{0}\right)=0$, we know that $X_{0}$ is the equilibrium of the system (26). On the other hand, it can be seen that $H(X)$ has a local strict minimum at $X_{0}$. From the properties of dissipative $\mathrm{PCH}$ systems, the system (26) is asymptotically stable at $X_{0}$, which means that $x \rightarrow x_{0}, \xi \rightarrow \xi_{0}$. Therefore, under the feedback controller (24), the systems (20) and (21) are simultaneously stabilized. 


\subsection{Adaptive Simultaneous Stabilization}

When systems (20) and (21) involve parametric uncertainties in their Hamiltonian structures, their dynamics become

$$
\begin{aligned}
& \left\{\begin{array}{l}
\dot{x}=\left[J_{1}\left(x, p_{1}\right)-R_{1}\left(x, p_{1}\right)\right] \nabla H_{1}\left(x, p_{1}\right)+g_{1}(x) u, \\
y_{1}=g_{1}^{T}(x) \nabla H_{1}(x),
\end{array}\right. \\
& \left\{\begin{array}{c}
\dot{\xi}=\left[J_{1}\left(\xi, p_{2}\right)-R_{2}\left(\xi, p_{2}\right)\right] \nabla H_{2}\left(\xi, p_{2}\right)+g_{2}(\xi) u, \\
y_{2}=g_{2}^{T}(\xi) \nabla H_{1}(\xi),
\end{array}\right.
\end{aligned}
$$

where $p_{1}, p_{2}$ are unknown vectors denoting the parametric uncertainties of the two Hamiltonian structures, respectively; for simplicity, we still denote the structural matrices by $J_{i}$ and $R_{i}$, and when $p_{i}=0, J_{i}(x, 0)=J_{i}(x), R_{i}(x, 0)=R_{i}(x)$ and $H_{i}(x, 0)=H_{i}(x), i=1,2 ; p_{1}$ and $p_{2}$ are assumed to be small enough to keep the two dissipative Hamiltonian structures unchanged, $J_{i}^{T}\left(x, p_{i}\right)=-J_{i}\left(x, p_{i}\right)$ and $R_{i}\left(x, p_{i}\right) \geq 0, i=1,2$. For further details, please see [14,20]. Now, we present an adaptive simultaneous stabilization result for systems (30) and (31).

For the adaptive simultaneous stabilization problem of systems (30) and (31), we have the following results.

\section{Theorem 2. Assume that}

(i) there exists a symmetric matrix $K \in \mathbb{R}^{6 \times 6}$ such that

$$
\left\{\begin{array}{l}
\bar{R}_{1}\left(x, p_{1}\right):=R_{1}\left(x, p_{1}\right)+K_{11}(x, x)>0 \\
\bar{R}_{2}\left(\xi, p_{2}\right):=R_{2}\left(\xi, p_{2}\right)-K_{22}(\xi, \xi)>0
\end{array}\right.
$$

where $K_{i j}(x, \xi)=g_{i}(x) K g_{j}^{T}(\xi),(i, j=1,2)$;

(ii) there exists a matrix $\Phi$ with appropriate dimension such that

$$
\left[J_{i}\left(x, p_{i}\right)-R_{i}\left(x, p_{i}\right)\right] \triangle_{H_{i}\left(x, p_{i}\right)}=g_{i}(x) \Phi \theta,(i=1,2),
$$

where $\triangle_{H_{i}\left(x, p_{i}\right)}=\nabla H_{i}\left(x, p_{i}\right)-\nabla H_{i}(x), \theta$ is an unknown constant vector related to $p_{1}$ and $p_{2}$.

Then, under the following adaptive feedback law:

$$
\left\{\begin{array}{l}
u=-K\left(y_{1}-y_{2}\right)-\Phi \widehat{\theta} \\
\dot{\hat{\theta}}=Q \Phi^{T}\left(y_{1}+y_{2}\right)
\end{array}\right.
$$

the systems (30) and (31) can be simultaneously stabilized, where $\widehat{\theta}$ is the estimate of $\theta$ and $Q>0$ is a positive definite constant matrix called the adaptation gain matrix.

Proof. Substituting (34) into systems (30) and (31) and using condition (ii), we obtain

$$
\left\{\begin{aligned}
\dot{x}= & {\left[J_{1}\left(x, p_{1}\right)-R_{1}\left(x, p_{1}\right)\right] \nabla H_{1}(x)-K_{11}(x, x) \nabla H_{1}(x) } \\
& +K_{12}(x, \xi) \nabla H_{2}(\xi)+g_{1}(x) \Phi(\theta-\widehat{\theta}), \\
\dot{\xi}= & {\left[J_{2}\left(\xi, p_{2}\right)-R_{2}\left(\xi, p_{2}\right)\right] \nabla H_{2}(\xi)-K_{21}(\xi, x) \nabla H_{1}(x) } \\
& +K_{22}(\xi, \xi) \nabla H_{2}(\xi)+g_{2}(\xi) \Phi(\theta-\widehat{\theta}), \\
\dot{\hat{\theta}}= & Q \Phi^{T}\left(g_{1}^{T}(x) \nabla H_{1}(x)+g_{2}^{T}(\xi) \nabla H_{2}(\xi)\right) .
\end{aligned}\right.
$$


Furthermore, the system (35) can be written as

$$
\left[\begin{array}{c}
\dot{x} \\
\dot{\xi} \\
\dot{\hat{\theta}}
\end{array}\right]=\left[\begin{array}{ccc}
J_{1}\left(x, p_{1}\right)-\bar{R}_{1}\left(x, p_{1}\right) & K_{12}(x, \xi) & -g_{1}(x) \Phi Q \\
-K_{21}(\xi, x) & J_{2}\left(\xi, p_{2}\right)-\bar{R}_{2}\left(\xi, p_{2}\right) & -g_{2}(\xi) \Phi Q \\
Q \Phi^{T} g_{1}^{T}(x) & Q \Phi^{T} g_{2}^{T}(\xi) & 0
\end{array}\right]\left[\begin{array}{c}
\nabla H_{1}(x) \\
\nabla H_{2}(\xi) \\
-Q^{-1}(\theta-\widehat{\theta})
\end{array}\right],
$$

letting $X:=\left[x^{T}, \xi^{T}, \widehat{\theta}^{T}\right]^{T}$,

$$
\begin{gathered}
J(X, p)=\left[\begin{array}{ccc}
J_{1}\left(x, p_{1}\right) & K_{12}(x, \xi) & -g_{1}(x) \Phi Q \\
-K_{21}(\xi, x) & J_{2}\left(\xi, p_{2}\right) & -g_{2}(\xi) \Phi Q \\
\left(g_{1}(x) \Phi Q\right)^{T} & \left(g_{2}(\xi) \Phi Q\right)^{T} & 0
\end{array}\right], \\
R(X, p)=\left[\begin{array}{ccc}
\bar{R}_{1}\left(x, p_{1}\right) & 0 & 0 \\
0 & \bar{R}_{2}\left(\xi, p_{2}\right) & 0 \\
0 & 0 & 0
\end{array}\right], \\
H(X):=H_{1}(x)+H_{2}(\xi)+\frac{1}{2}(\theta-\widehat{\theta})^{T} Q^{-1}(\theta-\widehat{\theta}),
\end{gathered}
$$

and $p=\left[p_{1}^{T}, p_{2}^{T}\right]^{T}$, then the system (36) can be expressed as

$$
\dot{X}=[J(X, p)-R(X, p)] \nabla H(X) .
$$

Notice that $K_{12}^{T}(x, \xi)=K_{21}(\xi, x), J(X, p)$ is a skew matrix. From condition (i), $R(X, p)$ is positive semidefinite. Thus, system (40) is a dissipative Hamiltonian system. Known by the characteristics of the dissipative Hamiltonian system, we know that system (40) is stable.

\subsection{Adaptive Robust Simultaneous Stabilization}

In the subsection, on the basis of the above simultaneous stabilization result, we study the adaptive robust simultaneous stabilization problem for systems (30) and (31) with external disturbances and uncertainties.

To do this, consider the systems (30) and (31) subject to external interference as

$$
\begin{aligned}
& \left\{\begin{aligned}
\dot{x} & =\left[J_{1}\left(x, p_{1}\right)-R_{1}\left(x, p_{1}\right)\right] \nabla H_{1}\left(x, p_{1}\right)+g_{1}(x) u+\bar{g}_{1}(x) \omega, \\
y_{1} & =g_{1}^{T}(x) \nabla H_{1}(x),
\end{aligned}\right. \\
& \left\{\begin{array}{c}
\dot{\xi}=\left[J_{1}\left(\xi, p_{2}\right)-R_{2}\left(\xi, p_{2}\right)\right] \nabla H_{2}\left(\xi, p_{2}\right)+g_{2}(\xi) u+\bar{g}_{2}(\xi) \omega, \\
y_{2}=g_{2}^{T}(\xi) \nabla H_{1}(\xi),
\end{array}\right.
\end{aligned}
$$

where $p_{1}, p_{2}$ are unknown vectors denoting the parametric uncertainties of the two Hamiltonian structures, respectively, $\omega$ is external interference, $J_{i}(x, 0)=J_{i}(x), R_{i}(x, 0)=R_{i}(x)$ and $H_{i}(x, 0)=$ $H_{i}(x), i=1,2, p_{1}$ and $p_{2}$ are assumed to be small enough to keep the two dissipative Hamiltonian structures unchanged. That is, $p_{1}, p_{2}$ are satisfied $J_{i}^{T}\left(x, p_{i}\right)=-J_{i}\left(x, p_{i}\right)$ and $R_{i}\left(x, p_{i}\right) \geq 0, i=1,2$.

Given a disturbance attenuation level $\gamma>0$, choose

$$
z=\Lambda\left(y_{1}+y_{2}\right)
$$

as the penalty function, where $\Lambda$ is a weight matrix with an appropriate dimension. The aim of this subsection is described as follows: 
We design an output feedback $L_{2}$ interference suppression controller, so that the following requirements are met under the action of the controller:

(a) When $\omega=0$, system (41) and system (42) can be simultaneously stabilized;

(b) The $L_{2}$ gain (from $\omega$ to $z$ ) of the closed-loop system is not greater than the given $\gamma$.

For the robust adaptive simultaneous stabilization problem, we have the following result.

Theorem 3. Assume that

(i) there exists a symmetric matrix $K \in \mathbb{R}^{6 \times 6}$ such that

$$
\left\{\begin{array}{l}
\bar{R}_{1}\left(x, p_{1}\right):=R_{1}\left(x, p_{1}\right)+K_{11}(x, x)>0, \\
\bar{R}_{2}\left(\xi, p_{2}\right):=R_{2}\left(\xi, p_{2}\right)-K_{22}(\xi, \xi)>0
\end{array}\right.
$$

where $K_{i j}(x, \xi)=g_{i}(x) K g_{j}^{T}(\xi),(i, j=1,2)$;

(ii) there exists a matrix $\Phi$ with appropriate dimension such that

$$
\left[J_{i}\left(x, p_{i}\right)-R_{i}\left(x, p_{i}\right)\right] \triangle_{H_{i}\left(x, p_{i}\right)}=g_{i}(x) \Phi \theta,(i=1,2),
$$

where $\triangle_{H_{i}\left(x, p_{i}\right)}=\nabla H_{i}\left(x, p_{i}\right)-\nabla H_{i}(x), \theta$ is an unknown vector related to $p_{1}$ and $p_{2}$;

$$
\text { (iii) } \begin{aligned}
\bar{g}_{1}=g_{1}= & {\left[\begin{array}{c}
0 \\
M_{1}^{-1}
\end{array}\right], \bar{g}_{2}=g_{2}=\left[\begin{array}{c}
0 \\
M_{2}^{-1}
\end{array}\right], \text { then } } \\
& \left\{\begin{array}{l}
u=-K\left(y_{1}-y_{2}\right)-\left[\frac{1}{2} \Lambda^{T} \Lambda+\frac{1}{2 \gamma^{2}} I_{6}\right]\left(y_{1}+y_{2}\right)-\Phi \widehat{\theta}, \\
\hat{\theta}=Q \Phi^{T}\left(y_{1}+y_{2}\right),
\end{array}\right.
\end{aligned}
$$

is an adaptive robust simultaneous stabilization controller such that both (a) and (b) hold true for the systems (41) and (42), where $\hat{\theta}$ is the estimate of $\theta$ and $Q>0$ is a positive definite constant matrix.

Proof. We rewrite (46) as follows:

$$
\left\{\begin{array}{l}
\left\{\begin{array}{l}
u=-K\left(y_{1}-y_{2}\right)-\Phi \widehat{\theta}+v, \\
\hat{\theta}=Q \Phi^{T}\left(y_{1}+y_{2}\right)
\end{array}\right. \\
v=-\left[\frac{1}{2} \Lambda^{T} \Lambda+\frac{1}{2 \gamma^{2}} I_{6}\right]\left(y_{1}+y_{2}\right) .
\end{array}\right.
$$

Substituting the first part of (47) into the systems (41) and (42), from the proof of Theorem 2 and condition (ii), the systems (41) and (42) are expressed as

$$
\dot{X}=[J(X, p)-R(X, p)] \nabla H(X)+G(X) v+\bar{G}(X) \omega,
$$

where $X:=\left[x^{T}, \xi^{T}, \widehat{\theta}^{T}\right]^{T}, \quad p=\left[p_{1}^{T}, p_{2}^{T}\right]^{T}$,

$$
\begin{gathered}
J(X, p)=\left[\begin{array}{ccc}
J_{1}\left(x, p_{1}\right) & K_{12}(x, \xi) & -g_{1}(x) \Phi Q \\
-K_{12}(x, \xi) & J_{2}\left(\xi, p_{2}\right) & -g_{2}(\xi) \Phi Q \\
\left(g_{1}(x) \Phi Q\right)^{T} & \left(g_{2}(\xi) \Phi Q\right)^{T} & 0
\end{array}\right], \\
R(X, p)=\left[\begin{array}{ccc}
\bar{R}_{1}\left(x, p_{1}\right) & 0 & 0 \\
0 & \bar{R}_{2}\left(\xi, p_{2}\right) & 0 \\
0 & 0 & 0
\end{array}\right],
\end{gathered}
$$




$$
H(X):=H_{1}(x)+H_{2}(\tilde{\xi})+\frac{1}{2}(\theta-\widehat{\theta})^{T} Q^{-1}(\theta-\widehat{\theta}),
$$

$G(X):=\left[g_{1}^{T}(x), g_{2}^{T}(\xi), 0\right]^{T}, \bar{G}(X):=\left[\bar{g}_{1}^{T}(x), \bar{g}_{2}^{T}(\xi), 0\right]^{T}$.

On the other hand, the penalty function (43) can be rewritten as

$$
z=\Lambda G^{T}(X) \nabla H(X)
$$

Furthermore, from conditions (i) and (iii), one can obtain

$$
R(X, p)+\frac{1}{2 \gamma^{2}}\left[G(X) G^{T}(X)-\bar{G}(X) \bar{G}^{T}(X)\right]=R(X, p) \geq 0 .
$$

Therefore, the system satisfies all the conditions of Lemma 1. From Lemma 1, the $L_{2}$ disturbance attenuation controller for the system (48) can be designed as

$$
v=-\left[\frac{1}{2} \Lambda^{T} \Lambda+\frac{1}{2 \gamma^{2}} I_{6}\right] G^{T}(X) \nabla H(X)=-\left[\frac{1}{2} \Lambda^{T} \Lambda+\frac{1}{2 \gamma^{2}} I_{6}\right]\left(y_{1}+y_{2}\right),
$$

and furthermore, the $\gamma$ - dissipation inequality

$$
\dot{H}+\nabla^{T} H(X) R(X, p) \nabla H(X) \leq \frac{1}{2}\left\{\gamma^{2}\|\omega\|^{2}-\|z\|^{2}\right\}
$$

holds along the trajectories of the closed-loop system consisting of (48) and (54), where $Q(x)=$ $\nabla^{T} H(X) R(X, p) \nabla H(X)$.

Noticing that (54) is the second part of (47), and by the characteristic P1 in Remark 1, it is easy to obtain that (b) is established. On the other hand, from (55), we know that when $\omega=0$,

$$
\begin{aligned}
\dot{H} & \leq-\nabla^{T} H(X) R(X, p) \nabla H(X) \\
& =-\nabla^{T} H_{1}(x) \bar{R}_{1}\left(x, p_{1}\right) \nabla H_{1}(x)-\nabla^{T} H_{2}(\xi) \bar{R}_{2}\left(\xi, p_{2}\right) \nabla H_{2}(\xi) \leq 0
\end{aligned}
$$

with which it is easy to see that the closed-loop system consisting of (48) and (54) converges to the largest invariant set contained in

$$
S:=\left\{X \mid \bar{R}_{1}^{\frac{1}{2}}\left(x, p_{1}\right) \nabla H_{1}(x)=0, \bar{R}_{2}^{\frac{1}{2}}\left(\xi, p_{2}\right) \nabla H_{1}(\xi)=0\right\},
$$

from condition (i), $\bar{R}_{1}^{\frac{1}{2}}\left(x, p_{1}\right) \nabla H_{1}(x)=0 \Rightarrow x=x_{0}, \bar{R}_{2}^{\frac{1}{2}}\left(\xi, p_{2}\right) \nabla H_{1}(\xi)=0 \Rightarrow \xi=\xi_{0}$. Therefore, $x \rightarrow x_{0}$ and $\xi \rightarrow \xi_{0}($ as $t \rightarrow \infty)$, which means that (a) also holds under the feedback (46). The proof is completed.

Remark 2. Since the adaptation considered in this paper is the perturbation of the internal structural parameters of the system, corresponding to the ship model, we assume the inertia matrix $M=M_{0}+M_{\delta}$, where $M_{0}$ is the normal part and $M_{\delta}$ is the unknown part. Using condition (ii) in Theorem 3 and combined with [21], we obtain

$$
\left[\triangle J_{i}\left(x, p_{i}\right)-\triangle R_{i}\left(x, p_{i}\right)\right] \triangle_{H_{i}\left(x, p_{i}\right)}=\left[\begin{array}{cc}
0 & 0 \\
0 & M_{\delta i}^{-1} D_{i} x_{2}
\end{array}\right]=g_{i}(x)\left[\begin{array}{ll}
0 & M_{i} x_{2}
\end{array}\right] M_{\delta i}^{-1} D_{i}
$$

where $\Phi_{i}=\left[\begin{array}{ll}0 & M_{i} x_{2}\end{array}\right], \theta_{i}=M_{\delta i} D_{i}, i=1,2$. Then $\Phi=\left[\Phi_{1}, \Phi_{2}\right]^{T}=\left[\begin{array}{ll}0 & M_{1} x_{2} \\ 0 & M_{2} \xi_{2}\end{array}\right], \theta=\left[\theta_{1}, \theta_{2}\right]^{T}=$ $\left[M_{\delta 1} D_{1}, M_{\delta 2} D_{2}\right]^{T}$. Therefore, the conditions (ii) of Theorems 2 and 3 are reasonable for the ship system. 


\section{Simulation}

In this section, we use simulink to simulate the two supply ships of Northern Clipper to further verify the effectiveness of the designed controller. The mass matrix and damping matrix of the supply ships Northern Clipper are as follows [22]:

$$
\begin{gathered}
M=\left[\begin{array}{ccc}
5.3122 \times 10^{6} & 0 & 0 \\
0 & 8.2831 \times 10^{6} & 0 \\
0 & 0 & 3.7454 \times 10^{9}
\end{array}\right] \\
D=\left[\begin{array}{ccc}
5.0242 \times 10^{4} & 0 & 0 \\
0 & 2.7299 \times 10^{5} & -4.3933 \times 10^{6} \\
0 & -4.3933 \times 10^{6} & 4.1894 \times 10^{8}
\end{array}\right] .
\end{gathered}
$$

The initial positions of the two ships are $\left(10 \mathrm{~m}, 5 \mathrm{~m}, 0^{\circ}\right),\left(15 \mathrm{~m}, 10 \mathrm{~m}, 0^{\circ}\right)$ and the initial speeds are $(1 \mathrm{~m} / \mathrm{s}, 0.8 \mathrm{~m} / \mathrm{s}, 2 \mathrm{rad} / \mathrm{s}),(1.2 \mathrm{~m} / \mathrm{s}, 1 \mathrm{~m} / \mathrm{s}, 3 \mathrm{rad} / \mathrm{s})$ respectively. The parameters of the proposed controller are chosen as $K=\operatorname{Diag}\{1,-1,2,1,-1,2\}, k_{1}=\operatorname{Diag}\{0.5,0.5,0.5\}, \Lambda=\operatorname{Diag}\{0.2,0.3,0.4\}$, $\gamma=0.4, M_{\delta}=0.2 M, Q=1$. In this article, the interference we designed in the simulation is assumed to add a common square wave with an amplitude of 1 within 1-2 s. The simulation results are shown in Figures 1-6. Among them, Figures 1-3 are the positional state curves of the two ships respectively, and Figures 4-6 are the speed state curves of the two ships respectively.

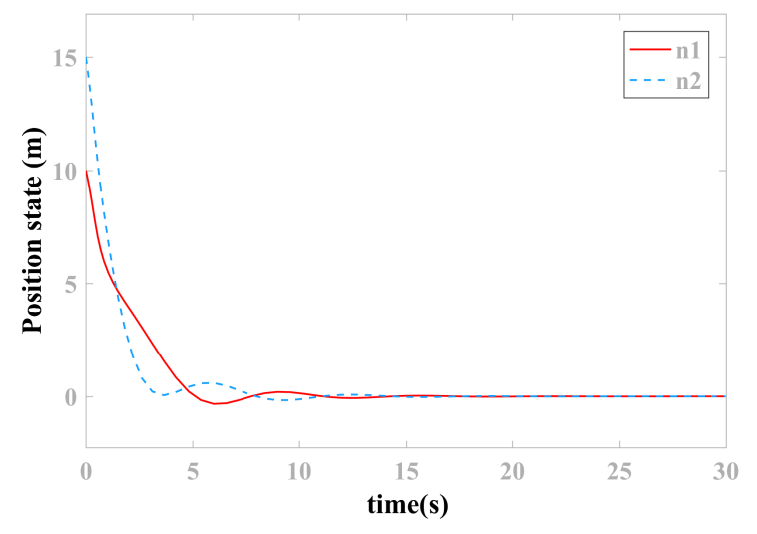

Figure 1. Surge displacement state of two ships $(n 1, n 2)$.

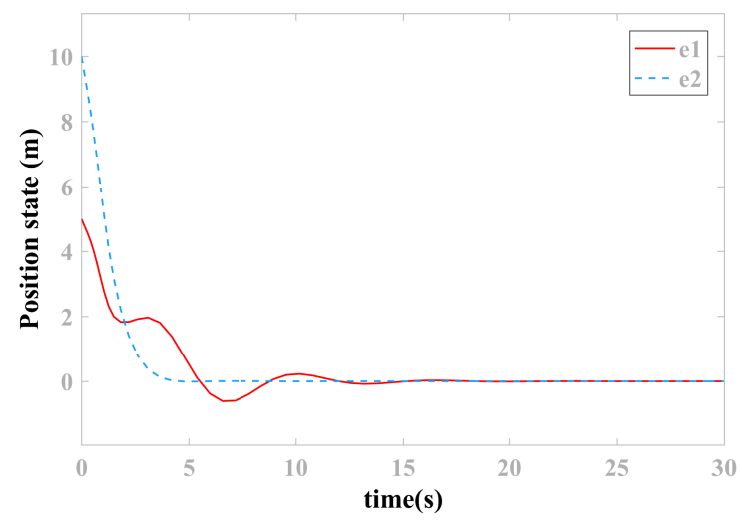

Figure 2. Sway displacement state of two ships $(e 1, e 2)$. 


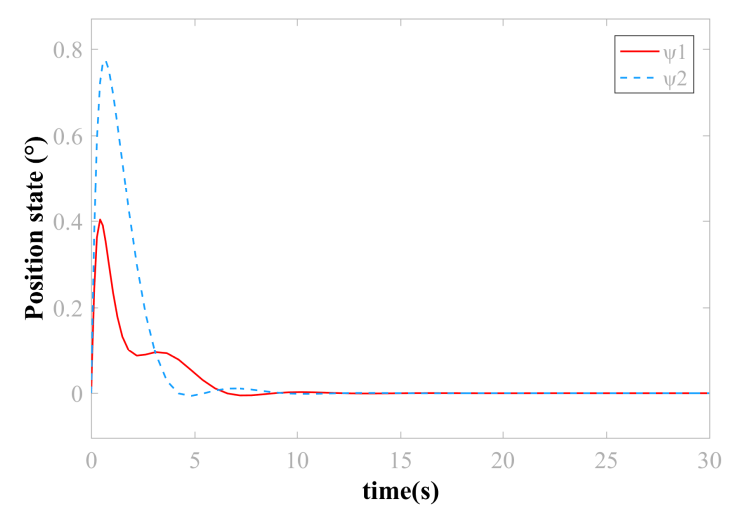

Figure 3. Yaw angle state of two ships $(\psi 1, \psi 2)$.

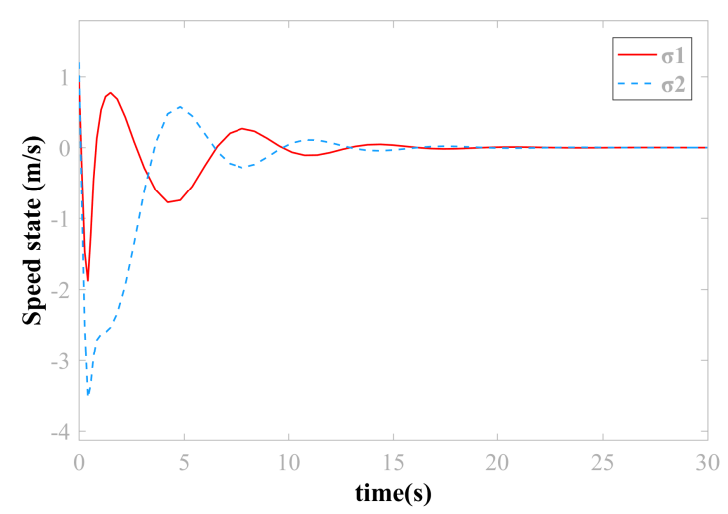

Figure 4. Surge speed state of two ships $(\sigma 1, \sigma 2)$.

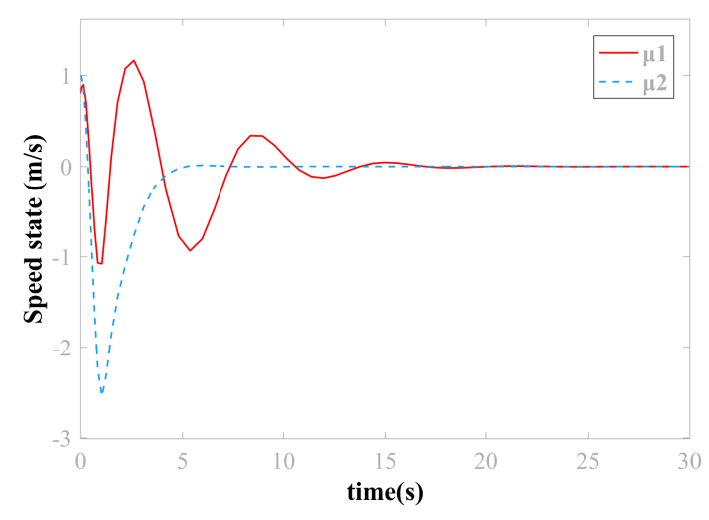

Figure 5. Sway speed state of two ships $(\mu 1, \mu 2)$.

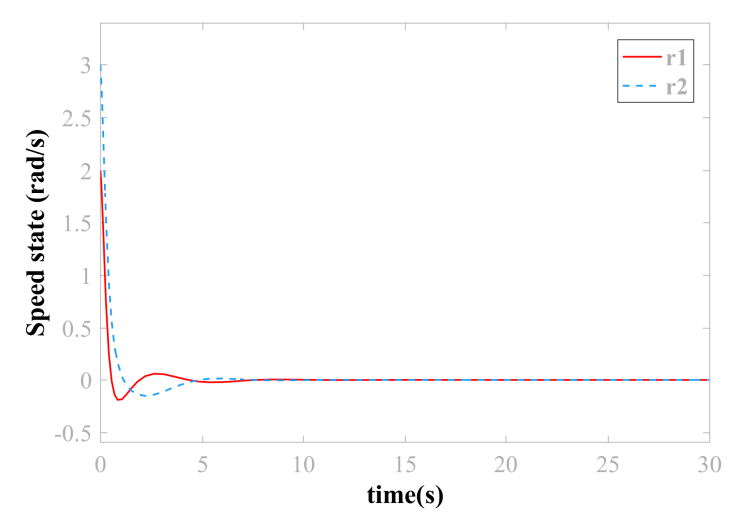

Figure 6. Yaw angrular speed state of two ships $(r 1, r 2)$. 
From Figures 1-6, we know that the designed robust adaptive control law can overcome the environmental interference force and gradually tend to the simultaneous stabilization of the ships. During the process of movement, the expecting heading angle is firstly reached in a short time, then the expecting longitudinal displacement is reached, and finally the lateral propulsion gradually reaches the expecting position and remains calm. Under the action of the controller, the ships gradually converge to the anchor point, and the position and the heading yaw direction tend to stabilize within $25 \mathrm{~s}$.

\section{Conclusions}

The paper has investigated the robust simultaneous stabilization problem of two identical supply ships, by converting the water surface three-degree-of-freedom ship model into a port-controlled model. Based on the Hamilton method, we firstly designed a simultaneous stabilization controller and an adaptive simultaneous stabilization controller for the systems without the disturbance, and then we studied the adaptive robust simultaneous stabilization problem on the system with the external disturbance, and designed an output feedback controller. In contrast to the existing literature, this paper used the Hamiltonian method and dimensional expansion technology, to obtain concise results. Our simulation results show the effectiveness of the designed controller in the paper.

Author Contributions: Conceptualization, P.Z. and R.Y.; methodology, R.Y., G.Z. and Y.H.; formula derivation, P.Z. and R.Y.; resources, G.Z. and Y.H.; simulation, P.Z.; writing-review and editing, P.Z. and R.Y.; project administration, R.Y. and Y.H.; funding acquisition, G.Z.

Funding: This work was partially supported by the National Natural Science Foundation of China (G61773015, G61877028, G61803230), Shandong Province Key Research and development Project (2018GGX105003, 2018GGX105014), Natural Science Foundation of Shandong Province (ZR2019MF032), A Project of Shandong Province Higher Educational Science and Technology Program (J18KA330), and Implementation Plan of "1251" Talent Training Project of Shandong Jiaotong University, University outstanding youth innovation team development plan of Shandong Province under Grant 2019KJN023.

Conflicts of Interest: The authors declare no conflict of interest.

\section{References}

1. Bian, X.; Fu, M.; Wang, Y. Ship Dynamic Positioning; Science Press: Beijing, China, 2011; pp. 1-13.

2. Ding, X. Ship Dynamic Positioning Control System; Dalian Maritime University Press: Dalian, China, 2017; pp. 1-30.

3. Jiao, J.; Wang, G.; Gu, Y.; Sun, X. Coordinated Formation Control of Dynamically Positioned Ships; Electronic Industry Press: Beijing, China, 2017; pp. 1-20.

4. Wang, B. Robust Adaptive Formation Control for Multiple Dynamic Positioning Vessels. Ph.D. Thesis, Harbin Engineering University, Harbin, China, 2017.

5. Wang, S. Research on Consistency Theory of Multi-Dynamic Positioning Vessels. Ph.D. Thesis, Harbin Engineering University, Harbin, China, 2014.

6. Li, Y.; Xu, F.; Xie, G. Overview of the development and application of multi-agent technology. Comput. Eng. Appl. 2018, 54, 13-21.

7. $\mathrm{Xu}, \mathrm{G}$. Research on Unified Multi-Agent System and Formation Control Design. Ph.D. Thesis, Taiyuan University, Taiyuan, China, 2018.

8. Chen, G. Study on the Stabilization Control of Underactuated Surface Vessels. Ph.D. Thesis, Dalian Maritime University, Dalian, China, 2014.

9. Kang, Z.; Zou, J.; Fang, L. Design of a motion stabilization controller for a fully driven ship. SHIP BOAT 2018, 29, 51-58.

10. Muhammad, S.; DoRia-Cerezo, A. Passivity-based control applied to the dynamic positioning of ships. IET Control Theory Appl. 2012, 6, 680-688. [CrossRef]

11. Tu, F.; Ge, S.; Choo, Y.S.; Hang, C.C. Adaptive Dynamic Positioning Control for Accommodation Vessels with Multiple Constraints. IET Control Theory Appl. 2016, 11, 329-340. [CrossRef]

12. Mao, J. Simultaneous Stabilization and Simultaneous $H \infty$ Control for Uncertain Nonlinear Systems. Ph.D. Thesis, Zhengzhou University, Zhengzhou, China, 2011. 
13. Cai, X.; Gao, H.; Liu, Y. Simultaneous $H \infty$ Stabilization for a Class of Multi-input Nonlinear Systems. Acta Autom. Sin. 2012, 38, 473-478. [CrossRef]

14. Wang, Y.; Liu, Y.; Gang, F. Simultaneous stabilization of nonlinear port-controlled Hamiltonian systems via output feedback. J. Shandong Univ. Eng. Sci. 2009, 39, 52-63.

15. Zhang, J. Simultaneous Stabilization and Control Stydy of Time-delay Systems. Ph.D. Thesis, Inner Mongolia Normal University, Hohhot, China, 2016.

16. Li, R. Simultaneous Stabilization and Control for Singular Systems with Time-delay. Ph.D. Thesis, Inner Mongolia Normal University, Hohhot, China, 2016.

17. Liang, Y. Simultaneous Stabilization and Control for Inter-connected Large-scale System with Time-delay. Ph.D. Thesis, Inner Mongolia Normal University, Hohhot, China, 2016.

18. Wang, Y. Generalized Hamiltonian Control System Theory: Implementation, Control and Application; Science Press: Beijing, China, 2017.

19. Fossen T.I. Handbook of Marine Craft Hydrodynamics and Motion Control; John Wiley and Sons Ltd. Press: West Sussex, UK, 2011; pp. 45-58.

20. Wang, Y.; Gang, F.; Cheng, D. Simultaneous stabilization of a set of nonlinear port-controlled Hamiltonian systems. Automatica 2007, 43, 403-415. [CrossRef]

21. Yang, R.; Guo, R. Adaptive finite-time robust control of nonlinear delay Hamiltonian systems via Lyapunov-Krasovskii method. Asian J. Control 2018, 20, 1-11. [CrossRef]

22. Hu, X. Reserch on Nonlinear Control for Dynamic Positioning of Ships. Ph.D. Thesis, Dalian Maritime University, Dalian, China, 2018.

(C) 2019 by the authors. Licensee MDPI, Basel, Switzerland. This article is an open access article distributed under the terms and conditions of the Creative Commons Attribution (CC BY) license (http:/ / creativecommons.org/licenses/by/4.0/). 